\title{
Über den Zuckerstoffwechsel der nervösen Zentralorgane.
}

\author{
Von \\ Else Hirschberg und Hans Winterstein. \\ (Aus dem physiologischen Institut der Universität Rostock.) \\ (Der Redaktion zugegangen am 4. Juli 1917.)
}

I. Versuche am isolierten Froschrückenmark.

Das Bestehen eines intensiven respiratorischen Gaswechels, über den wir durch eine Reihe hauptsächlich aus dem hiesigen Institute hervorgegangener Arbeiten einigermaßen orientiert sind, und die Tatsache einer Säurebildung bei unzureichender Sauerstoffversorgung ist das einzige, was wir bisher über den Stoffwechsel der nervösen Zentralorgane wissen. Ein Versuch, unsere Kenntnisse hierüber an höheren Tieren und unter den normalen Bedingungen der Blutversorgung zu erweitern, begegnet aus naheliegenden Gründen vorläufig wohl unüberwindlichen Schwierigkeiten; das isolierte Froschrückenmark, dessen Überlebensbedingungen uns durch zahlreiche Untersuchungen genügend bekannt sind, bleibt zunächst das einzige Objekt, das eine weitere Verfolgung dieser von verschiedenen Gesichtspunkten aus sicher sehr bedeutungsvollen Probleme ermöglicht. Die neueren analytischen Methoden sind zum Teil ausreichend genau, um trotz der Kleinheit des Organs eine Inangriffnahme anderweitiger Stoff wechselversuche nicht aussichtslos erscheinen $\mathrm{zu}$ lassen. Und so soll in der folgenden Mitteilung zunächst die Frage untersucht werden, ob und in welchem Umfange unter verschiedenen Bedingungen das isolierte Froschrückenmark Traubenzucker in der umgebenden Lösung umzusetzen vermag.

Methodik. Die Versuche wurden in der Weise angestellt, daß 2 oder 3 isolierte Froschrückenmarke für längere 
Zeit in eine kontinuierlich von Sauerstoff durchströmte Kochsalzlösung eingelegt wurden, die etwas Traubenzucker enthielt. Am Ende des Versuches wurde in Proben der Zuckergehalt sowohl der Lösung, in der das Organ verweilt hatte, wie der (gleichfalls mit Sauerstoff gesättigten) ursprünglichen Lösung nach der Bertrandschen Methode ${ }^{1}$ ) bestimmt, unter Verwendung von $\mathrm{n} / 100-\mathrm{KMnO}_{4}$ zur Titration. Aus der Differenz des Zuckergehaltes beider Lösungen ergab sich der Zuckerverbrauch, der auf $1 \mathrm{~g}$ Substanz und 24 Stunden umgerechnet wurde. Bei den (in weitaus den meisten Versuchen ausgeführten) Doppelbestimmungen der gleichen Lösung ergab sich als größte Differenz $0,05 \mathrm{ccm} \mathrm{KMnO}_{4}$. Da die ganze Differenz bei den Stoffwechselversuchen meist nur wenige Zehntel Kubikzentimeter ausmachte, so waren die absoluten Werte des Zuckerverbrauches allerdings einem beträchtlichen Fehler ausgesetzt, der unter Umständen 20 und mehr Prozent betragen konnte; die ausgezeichnete Übereinstimmung der Versuche untereinander aber beweist, daß die auf die Beeinflussung des Zuckerumsatzes durch die verschiedenen Faktoren gezogenen Schlußfolgerungen als absolut zuverlässig betrachtet werden können.

Einfluß der Gefäßhaut. Es zeigte sich, daß das in der gewöhnlichen Weise ${ }^{2}$ ) präparierte, von seiner Gefäßhaut (Pia mater + Arachnoidea) umhüllte Rückenmark innerhalb 24 Stunden eine zwar deutliche, aber doch nur sehr geringe Glykolyse aufweist. Nun wissen wir durch die Untersuchungen Ungers, ${ }^{3}$ ) daß zwar die Gewebselemente des Rückenmarks selbst für die verschiedensten Stoffe frei durchgängig zu sein scheinen, daß aber die Gefäßhaut merkwürdigerweise die Eigenschaften einer semipermeablen Membran besitzt, die man auf Grund der Untersuchungen am Froschmuskel als eine all-

1) Vgl. Abderhaldens Handb. d. biochem. Arbeitsmethoden, Bd. 2, S. 181.

2) Vgl. H. Winterstein, Über den Mechanismus der Gewebsatmung. Zeitschr. f. allg. Physiol., Bd. 6, S. 315 (1907).

$\left.{ }^{3}\right)$ R. Un ger, Untersuchungen über den Einfluß von anorganischen Lösungen auf die Oxydationsprozesse usw. Biochem. Zeitschr., Bd.61, S. 103 (1914). - Über physikalisch-chemische Eigenschaften des isolierten Froschrückenmarks und seiner Gefäßhaut. Ebenda, Bd. 80, S. 364 (1917). 
gemeine Eigenschaft aller Zellen aufzufassen geneigt war. Dies legte den Gedanken nahe, daß das Rückenmark unter den gegebenen Bedingungen aus rein äußeren Gründen nur wenig Zucker umzusetzen vermag, weil dieser an den von der Gefäßhaut umhüllten Teilen nicht oder nur in sehr geringem Maße eindringen kann und daher im wesentlichen bloß den freiliegenden Partien der Rautengrube und des Querschnittes durch das Kopfmark ausreichend zugänglich ist. Das Experiment bestätigte diese Vermutung in vollstem Umfange: Wurden nämlich zu den Versuchen Rückenmarke verwendet, bei denen die Gefäßhaut abgezogen worden war, so stieg der Zuckerverbrauch auf das Mehrfache. So betrug er bei Zimmertemperatur in zwei Versuchen mit zwei bezw. drei Rückenmarken + Gefäßhaut innerhalb der ersten 24 Stunden 1,2 mg pro $1 \mathrm{~g}$ Substanz, während er in einer Reihe von Versuchen mit piafreien Rückenmarken unter sonst gleichen Bedingungen 3,6-4,5 mg betrug. Noch deutlicher tritt der Einfluß der Gefäßhaut zutage, wenn man an einunddenselben Präparaten den Zuckerverbrauch vor und nach Entfernung der Pia untersucht. Hierzu ist es aber zunächst erforderlich, den EinfluB kennen zu lernen, den die Versuchsdauer auf die Größe der Glykolyse ausübt.

Wie von vornherein zu erwarten war, sinkt der Zuckerverbrauch mit der Versuchsdauer allmählich ab: Zwei (pialose) Rückenmarke, die innerhalb der ersten 24 Stunden einen Zuckerverbrauch von $4,5 \mathrm{mg}$ pro $1 \mathrm{~g}$ Substanz aufgewiesen hatten, verbrauchten am 2. Tage nurmehr $2,0 \mathrm{mg}$, und am 3 . Tage fiel der Zuckerverbrauch in die Fehlergrenzen der Methodik. Ein großes Froschrückenmark verbrauchte am 1. Tage $4 \mathrm{mg}$, am 2. Tage 2,4 mg pro $1 \mathrm{~g}$ Substanz. Der Versuchsdauer nach hätten mithin auch die ursprünglich in ihrer Gefäßhaut untersuchten Präparate nach Entfernung derselben eine Verminderung der Glykolyse zeigen müssen. In Wirklichkeit aber war gerade das Umgekehrte der Fall. Die drei Rückenmarke, die innerhalb der ersten 24 Stunden in ihrer Piahülle nur 1,2 mg pro $1 \mathrm{~g}$ umgesetzt hatten (s. o.), verbrauchten nach Entfernung derselben am 2. Tage $3,3 \mathrm{mg}$, und in ganz der 
gleichen Weise betrug der Zuckerverbrauch von 2 anderen Rückenmarken in ihrer Gefäßhaut am 1. Tage 1,2 mg, am 2. Tage nach Entfernung derselben $3,1 \mathrm{mg}$ pro $1 \mathrm{~g}$ Substanz; am 3. Tage war auch hier der Zuckerverbrauch so weit gesunken, daß er nicht mehr mit Sicherheit nachgewiesen werden konnte.

Die Feststellung des Einflusses, den die Gefäßhaut auf die Größe der Glykolyse ausübt, stellt einen neuen überzeugenden Beweis für die Richtigkeit der Ergebnisse dar, zu denen Unger (a. a. 0.) auf verschiedenen Wegen bezüglich der eigenartigen physikalisch-chemischen Eigenschaften dieses Gebildes gelangt ist. Der Umstand, daB die zunächst in ihrer Piaumhüllung untersuchten Rückenmarke nach Entfernung der Pia am 2. Tage einen größeren Zuckerverbrauch aufwiesen als die von vornherein der Pia beraubten $(3,3$ bezw. $3,1 \mathrm{mg}$ gegenüber 2,4 bezw. 2,0), dürfte seine Erklärung darin finden, daß die von Unger beschriebene Quellung des pialosen Rückenmarks in isotonischen Lösungen den Zuckerumsatz beeinträchtigt.

Diese Quellung verdient auch noch in anderer Hinsicht Berücksichtigung: $\mathrm{Zu}$ jedem Versuch wurden im allgemeinen 2 oder 3 Rückenmarke verwendet, deren Präparation 30-40 Minuten Zeit erforderte. Die zuerst entnommenen Organe wurden anfänglich bis zu der nach Beendigung der ganzen Präparation erfolgenden Wägung in physiologischer Kochsalzlösung aufbewahrt. Es zeigte sich, daß schon während dieser Zeit eine deutliche Wasseraufnahme stattfindet, sodaß sich ein zu großes Gewicht und bei der Umrechnung des Zuckerverbrauches auf die Gewichtseinheit daher ein etwas zu geringer Wert ergibt. In den späteren Versuchen wurde diese Fehlerquelle durch Aufbewahren der Rückenmarke in einer feuchten Kammer vermieden, in der auch die Wägung erfolgte. Auf dieses vor Beginn des Versuches ermittelte Anfangswicht wurden alle Daten bezogen.

Methodik des Abpräparierens der Gefäßhaut. Nachdem einmal der hindernde Einfluß der Gefäßhaut auf die Zuckerversorgung festgestellt war, wurden alle folgenden Versuche an pialosen Rückenmarken ausgeführt. Dies hat freilich außer der schon erwähnten Quellung den Nachteil, daß die Entfernung der Gefäßhaut nicht ohne erhebliche Schädigung des Organs zu bewerkstelligen ist, über deren Größe kein Aufschluß auf funktionellem Wege gewonnen werden kann, da mit der Gefäßhaut auch alle Nervenwurzeln entfernt werden, sodaß 
eine Untersuchung der Erregbarkeit nicht möglich ist. Immerhin werden die folgenden Versuche zeigen, daß diese Schädigung keine derartige ist, daß die die Funktion beeinflussenden Faktoren ihre Wirkung auf die Zuckerzersetzung verlieren würden. Die zuerst von Unger (a. a. 0.) ausgeführte Operation des Abziehens der Pia erfordert gewisse Übung, wenn sie sinigermaßen schonend erfolgen soll. Sucht man an dem in der gewöhnlichen Weise freigelegten Rückenmark die Gefäßhaut zu entfernen, so geht dies meist nicht ohne stellenweise Zerreißungen der weißen Substanz ab, da die Pinzetten keinen geeigneten Halt finden. Am besten hat sich schließlich das folgende Verfahren bewährt:

Der Schnitt zur Dekapitation des Frosches wird etwas höher als gewöhnlich geführt, sodaß er nicht an den oberen Rand des Kopfmarkes fällt, sondern noch einen Teil des Mittelhirns mit ihm in Verbindung läßt. Dann wird das Rückenmark durch Abtragung der Wirbelbogen von der dorsalen Seite freigelegt, der das Mittelhirn überdeckende hinterste Abschnitt der Schädelkapsel aber noch nicht entfernt, damit das Rückenmark an seinem obersten Ende festgehalten wird. Hierauf wird der unterste Teil desselben nach Entfernung der Dura mater mit einer Pinzette gefaßt und die Cauda equina berausgezogen, die nun als Handhabe für die weitere Präparation dient. Das an ihr leicht in die Höhe gehaltene Rückenmark wird durch von unten nach oben fortschreitende Durchschneidung aller Nervenwurzeln jetzt auch von der ventralen Seite freigelegt, sodaß es nurmehr mit dem Mittelhirnabschnitt am Körper festhaftet. Jetzt wird mit einer ganz feinen Pinzette die Gefäßhaut entfernt, indem die am weitesten nach unten gelegenen Nervenwurzeln gefaßst und in der Richtung nach dem Kopfende gezogen werden, wobei meist umfangreiche Abschnitte der Pia in einem Zuge sich loslösen. Nach Entfernung der Hauptteile werden die noch zurückgebliebenen kleineren Stücke sehr vorsichtig gefaßt und nach der einen oder anderen Richtung abgezogen, bis das Rückenmark in seiner ganzen Ausdehnung rein weiß erscheint. Erst dann wird der noch stehen gebliebene Teil der Schädelkapsel mit der Knochenzange abgetragen, das Rückenmark ganz herausgelöst und die am Kopfende noch anhaftenden Teile der Gefäßhaut ebenso wie die Mittelhirnreste entfernt.

Einfluß der Temperatur. $\mathrm{Zu}$ der ersten Reihe von Versuchen wurde $0,7 \%$ ige NaCl-Lösung verwendet, die $0,2 \%$ Traubenzucker enthielt. Wie schon oben erwähnt, sinkt in einer solchen der Zuckerverbrauch am zweiten Tage auf etwa die Hälfte $a b$ und ist am dritten so weit reduziert, daß er 
nicht mehr mit Sicherheit festgestellt werden kann, eine Beobachtung, die wohl mit der Tatsache in Einklang steht, daß auch die Erregbarkeit des isolierten Froschrückenmarks bei Zimmertemperatur nach längstens 48 Stunden erloschen $\mathrm{zu}$ sein pflegt (vgl. Winterstein, a. a. 0.). Dieses Absinken der Glykolyse mit der Versuchsdauer, sowie die Feststellung, daß die nach Entfernung der Rückenmarke zurückbleibende Lösung keine Zuckerzersetzung mehr zeigt, beweisen klar, daß ein Mitwirken irgendwelcher bakterieller Tätigkeit keine Rolle spielen kann.

Außer der Versuchsdauer ist weiter von Einfluß die Temperatur. Wie zu erwarten, steigt der Zuckerverbrauch mit Erhöhung der Temperatur, doch scheint der Temperaturkoeffizient nicht sehr groß zu sein. Während der Zuckerverbrauch in 5 Versuchen bei gewöhnlicher Temperatur $\left(13-18^{\circ}\right.$; innerhalb dieser Grenzen fällt der Einfluß der Temperatur in die individuellen Schwankungen) 3,6-4,5, im Mittel 4,0 mg pro $1 \mathrm{~g}$ Substanz und 24 Stunden betrug, verbrauchten zwei bei $4-6^{\circ}$ gehaltene Rückenmarke nur 2,9 , zwei bei $24^{\circ}$ gehaltene dagegen $6,3 \mathrm{mg}$ in Lösungen von gleichem Zuckergehalt.

Einfluß der Sauerstoff zufuhr. Um den Einfluß der Sauerstoffzufuhr festzustellen, wurde in einem Versuche durch die drei Rückenmarke enthaltende Lösung statt Sauerstoff während 8 Stunden Stickstoff (der einen ganz geringen Prozentsatz Sauerstoff enthielt) durchgeleitet. Der Zuckerverbrauch (bezogen auf $1 \mathrm{~g}$ und 24 Stunden) betrug nur $2,7 \mathrm{mg}$, zeigte aber auch bei nachfolgender. Sauerstoffzufuhr während 16 Stunden keine Steigerung mehr (2,2 mg pro $1 \mathrm{~g} \mathrm{u}$. 24 Stunden), wiederum in Einklang mit der Tatsache, daß auch die Erregbarkeit nach Erstickung nur wiederkehrt, wenn die Sauerstoffzufuhr rechtzeitig (nach etwa 2 Stunden) wiederhergestellt wird. Für den Zuckerverbrauch konnte ein derartiger Erholungsversuch nicht angestellt werden, weil die in 2 Stunden zersetzte Menge zu gering ist, um gemessen werden zu können.

Einfluß der Narkose. Eine größere Zahl von Experimenten wurde der Untersuchung solcher Faktoren gewidmet, 
welche die Erregbarkeit der Nervenzentren beeinflussen, um auf diese Weise mit Sicherheit feststellen zu können, daß es sich beim Zuckerverbrauch des überlebenden Rückenmarks um einen mit den funktionellen Lebensvorgängen auf das engste verknüpften Prozeß handelt. Solche Faktoren sind die Nark ose einerseits und die Reizung anderseits.

Als Narkotika dienten Äthylurethan und Äthylalkohol, deren Wirkung auf die Erregbarkeit und den Sauerstoffverbrauch des isolierten Froschrückenmarks von früheren Versuchen ${ }^{1}$ ) her genau bekannt war. Zur sicheren Feststellung ihres Einflusses wurde der Zuckerverbrauch an denselben Präparaten erst in Narkose und dann unter normalen Bedingungen untersucht. Zur Urethannarkose diente 0,7\% ige NaCl-Lösung, die außer dem gewöhnlichen Zuckergehalt noch einen solchen von 1 Vol.-Proz. Urethan aufwies. In zwei Versuchen wurden je zwei Rückenmarke erst 24 Stunden lang in dieser Lösung belassen und dann in die gewöhnliche Lösung übergeführt. Der Zuckerverbrauch betrug in Narkose 1,7 bezw. $1,5 \mathrm{mg}$ pro $1 \mathrm{~g}$ und während der darauffolgenden 24 Stunden unter normalen Bedingungen 2,8 bezw. $2,5 \mathrm{mg}$. Die Glykolyse war also trotz der langen Versuchsdauer nach der Narkose immer noch bedeutend höher als während der Narkose, in der sie unter Zugrundelegung des normalen Mittelwertes von $4 \mathrm{mg}$ eine Verminderung um rund $60 \%$ erfahren hatte. Noch stärker war die Herabsetzung unter dem Einfluß des Alkohols in der die Erregbarkeit aufhebenden Konzentration von 4 Vol.-Proz. Da der Alkohol viel schädlicher wirkt, wurden die Versuche nicht über so lange Zeit ausgedehnt, sondern in einem Versuche drei Rückenmarke erst 8 Stunden in der Alkohollösung und dann 16 Stunden unter gewöhnlichen Bedingungen, in einem zweiten Versuche zwei Rückenmarke erst 16 Stunden in der Alkohollösung und dann 8 Stunden in normaler Lösung untersucht. Im ersteren Falle sank der Zuckerverbrauch in den 8 Stunden unter die Grenze der Feststellbarkeit, im zweiten auf $2 / 3 \mathrm{mg}$ in den 16 Stunden (oder $1 \mathrm{mg}$ in 24 Stunden); die

1) H. Winterstein, Beiträge zur Kenntnis der Narkose. II. Mitteilung. Biochem. Zeitschr., Bd. 61, S. 81 (1914). 
in der darauffolgenden Erholung beobachteten Werte betrugen 2,5 bezw. 2,6 $\mathrm{mg}$ pro $1 \mathrm{~g}$ und 24 Stunden. - Es ist bemerkenswert, daß das Urethan, das nach früheren Erfahrungen (a. a. 0.) in der verwendeten Konzentration bloß die sensiblen und nicht die motorischen Mechanismen lähmt, den Zuckerverbrauch nicht so stark herabsetzt, wie $4 \%$ iger Alkohol, der die Funktionen beider aufhebt. Während so eine völlige Übereinstimmung in der Beeinflussung von Zuckerumsatz und Erregbarkeit besteht, gilt dies nicht in gleichem Maße für die Beeinflussung des Sauerstoffverbrauches, der unter der Einwirkung des Alkohols in der eben narkotischen Konzentration keine Herabsetzung, sondern sogar eine leichte Steigerung erfährt (vgl. Winterstein, a. a. 0.). Wir werden auf die Bedeutung dieser Erscheinung noch zurückkommen.

EinfluB der Reizung. Überzeugender noch als die Narkoseversuche demonstrieren die Reizversuche den innigen Zusammenhang zwischen Zuckerverbrauch und Nervenfunktion. Die beiden ersten Versuche wurden in der Weise angestellt, da $\beta$ in die gesamte Versuchszeit von 22 bezw. 24 Stunden eine $5^{1 / 2}-6$ stündige Reizungsperiode eingeschaltet wurde, während welcher durch die je zwei Rückenmarke enthaltende Lösung die Einzelschläge eines Induktionsapparates hindurchgeleitet wurden, dessen primärer Kreis durch eine Kontaktuhr $144 \mathrm{mal}$ in der Minute unterbrochen wurde. Das Ergebnis war ein Zuckerverbrauch von 6,0 bezw. 5,3 mg pro $1 \mathrm{~g}$ und 24 Stunden, also eine bedeutende Steigerung gegenüber dem normalen Mittelwerte von 4,0 und auch gegenüber dem höchsten unter den gleichen Bedingungen beobachteten Ruhewerte von $4,5 \mathrm{mg}$.

Viel drastischer aber gestalteten sich die folgenden Versuche, in denen der Zuckerverbraụch während der Ruhe- und während der Reizungsperiode gesondert an denselben Präparaten untersucht wurde, und zwar so, daß immer die Ruheperiode vorausging, soda $\beta$ die Versuchsdauer an sich nur eine Verminderung und nicht eine Steigerung des Zuckerumsatzes bewirken konnte. Zwei Rückenmarke von $140 \mathrm{mg}$ Gewicht, die während einer 15 stündigen Ruheperiode $0,37 \mathrm{mg}$ Zucker verbraucht hatten, verbrauchten in einer darauffolgenden 9 stün- 
digen Versuchszeit, innerhalb deren sie 5 Stunden hindurch mit 44 kurzen tetanisierenden Reizen in der Minute gereizt wurden, $0,50 \mathrm{mg}$, d. i. im ersteren Falle 4,2, im zweiten $9,6 \mathrm{mg}$ pro $1 \mathrm{~g}$ und 24 Stunden, oder eine Steigerung des Zuckerverbrauches um rund $130 \%$, ohne Berücksichtigung der durch die Versuchsdauer an sich etwa herbeigeführten Abnahme der Glykolyse. In der gleichen Weise verbrauchten zwei Rückenmarke von $197 \mathrm{mg}$ Gewicht in 16 stündiger Ruheperiode 0,47 , in der darauffolgenden 8 stündigen Reizungsperiode (35 kurzdauernde tetanisierende Reize pro Minute) $0,61 \mathrm{mg}$ oder auf $1 \mathrm{~g}$ und 24 Stunden berechnet 3,6 mg in der Ruhe und 9,2 mg während der Reizung, mithin eine Steigerung von über 155\%. Das gleiche Verhalten war in einem dritten mit $0,5 \%$ iger Zuckerlösung angestellten Versuche zu beobachten, in welchem der Zuckerverbrauch dreier $\mathbf{2 7 0}$ mg schwerer Rückenmarke während des 8 stündigen Reizungsversuches mit $0,99 \mathrm{mg}$ den in der vorangehenden 16 stündigen Ruheperiode festgestellten von 0,87 um $130 \%$ übertraf, indem er von 4,8 auf $11,0 \mathrm{mg}$ pro $1 \mathrm{~g}$ und 24 Stunden anstieg.

Die Tätigkeit der Nervenzentren bedingt mithin eine ganz außerordentliche Steigerung des Zuckerverbrauches, die das $1 \frac{1}{2}$ fache des Ruhewertes betragen kann und noch weit über die in früheren Versuchen') beobachtete Steigerung des Sauerstoffverbrauches bei Reizung hinausgeht.

Einfluß der Zuckerkonzentration. In dem letzterwähnten mit 0,5\% iger Zuckerlösung angestellten Reizversuche war nicht bloß der Reiz-, sondern auch der Ruhewert des Zuckerverbrauches beträehtlich höher als in allen vorangehenden Versuchen, in welchen eine 0,2\% ige Traubenzuckerlösung verwendet worden war. Dies veranlaßte uns, den Einfluß der Konzentration der Zuckerlösung auf die Größe des Zuckerumsatzes genauer zu untersuchen. Es ergab sich in der Tat, daß dieser ganz allgemein in einer 0,5\% igen Lösung höher war

1) Vgl. H. Winterstein, Der respiratorische Gaswechsel des isolierten Froschrückenmarks. Zentralblatt f. Physiol., Bd. 21, S. 869 (1908). 
als in einer $0,2 \%$ igen, wie besonders deutlich aus denjenigen Versuchen hervorgeht, in welchen die Glykolyse zuerst in 0,2und dann in $0,5 \%$ iger Lösung untersucht wurde. So betrug in einem Versuche der (stets auf $1 \mathrm{~g}$ und 24 Stunden berechnete) Zuckerverbrauch der Rückenmarke in 0,2\% iger Lösung in den ersten 14 Stunden $4,1 \mathrm{mg}$ und in den daraufolgenden 9 Stunden in $0,5 \%$ iger Lösung $5,5 \mathrm{mg}$ und in einem zweiten Versuche in 0,2\% iger Lösung während der ersten 8 Stunden 4,0 und in den darauffolgenden 15 Stunden in $0,5 \%$ iger Lösung $4,9 \mathrm{mg}$, zeigte also beide Male statt der sonst durch die Dauer des Versuches bedingten Abnahme eine Steigerung. Während sich in 0,2\% iger Lösung als Mittel von 7 Versuchen der Wert von $4,0 \mathrm{mg}(3,6-4,5)$ ergeben hatte, berechnete sich als Mittel von 7 mit 0,5\% iger Lösung angestellten Versuchen $5,1 \mathrm{mg}(4,8$ bis 5,5$)$.

War so der günstige Einfluß einer 0,5\% igen Zuckerlösung (die 0,6 oder $0,7 \% \mathrm{NaCl}$ enthielt) unzweifelhaft festgestellt, so konnte dagegen über den Einfluß noch höherer Konzentrationen keine völlige Klarheit gewonnen werden. Es zeigte sich, daß das Einbringen der Rückenmarke in eine 1 oder $2 \%$ Zucker enthaltende Lösung von unverändertem oder zur Wahrung der Isotonie entsprechend herabgesetztem NaCl-Gehalt die Glykolyse zunächst beträchtlich herabdrückte, daß diese aber nach längerem Verweilen wieder eine Erhöhung, mitunter sogar noch über den sonst in $0,5 \%$ iger Lösung beobachteten Wert erfahren konnte. Eine Erklärung für dieses merkwürdige Verhalten wurde nicht gefunden.

Einfluß des Calciums. Bei der Untersuchung des Einflusses verschiedener Ionen auf die Größe des Sauerstoffverbrauches des Froschrückenmarks hatte Unger ${ }^{1}$ ) den beträchtlichen oxydationshemmenden Einfluß des Calciums beobachtet, das bereits in einer Konzentration von $0,14 \%$ den Sauerstoffverbrauch in Ca-freier Lösung um fast $25 \%$ vermindern konnte. Ganz analog, ja, noch stärker ausgesprochen war die Wirkung des Calciums auf den Zuckerverbrauch, wie der folgende

1) Unger, Untersuchungen über den Einfluß von anorganischen Lösungen usw., a. a. 0. 
Versuch zeigt: Rückenmarkspräparate, die in einer $0,6 \% \mathrm{NaCl}$ und 0,5\% Zucker enthaltenden Lösung in den ersten 8 Stunden einen Zuckerbrauch von 5,2 mg (pro $1 \mathrm{~g}$ und 24 Stunden) aufgewiesen hatten, verbrauchten in den darauffolgenden 16 Stunden in der gleichen Lösung unter Zusatz von $0,1 \% \mathrm{CaCl}_{2}$ bloß $1,8 \mathrm{mg}$ und in den nächstfolgenden 21 Stunden wieder in der Ca-freien Lösung 2,6 mg (pro $1 \mathrm{~g}$ und 24 Stunden), mithin unter dem Einfluß des Calciums eine Herabsetzung des Wertes auf etwa 1/3, und nach Entfernung des Calciums wieder eine annähernde Rückkehr zur Norm, wenn man die am zweiten Versuchstage stets zu beobachtende Herabsetzung der Glykolyse in Rechnung zieht.

Einfluß des Natriums. Schon oben (vgl. S. 188) wurde erwähnt, daß die fortschreitende Quellung des Rückenmarks die allmähliche Abnahme des Zuckerumsatzes mitbedingen dürfte. Unger (a. a. 0) hatte beobachtet, daß der Sauerstoffverbrauch des pialosen Rückenmarks in destilliertem Wasser mit fortschreitender Quellung immer mehr absinkt, und hatte in seinen späteren Versuchen ${ }^{1}$ ) festgestellt, daß das $\mathrm{NaCl}$ einen sehr großen Einfluß auf die Quellung ausübt, die in salzfreier isotonischer Traubenzuckerlösung fast ebenso stark ist wie in destilliertem Wasser. In Einklang mit diesen Beobachtungen ergaben Versuche mit salzfreien Zuckerlösungen eine ganz außerordentliche Verminderung des Zuckerumsatzes, der in einem durch 21 Stunden fortgesetzten Versuch mit 1,2 mg pro $1 \mathrm{~g}$ und 24 Stunden nur eben die Grenze der Feststellbarkeit überschritt, und in einem zweiten nur 7 Stunden fortgesetzten Versuch zur Gänze unterhalb derselben blieb. Anderseits scheint auch ein zu hoher $\mathrm{NaCl}$-Gehalt schädigend zu wirken, da in einem Versuche mit Überführung der Präparate aus $0,6-$ in $2 \%$ ige Kochsalzlösung mit je $0,5 \%$ Zuckergehalt ein bedeutendes Absinken der Glykolyse feststellbar war.

Schließlich sei noch erwähnt, daß in einem Versuche die Wirkung eines Zusatzes von frischem Froschpankreasextrakt auf den Zuckerumsatz untersucht wurde, aber nach

1) Unger, Über physikalisch-chemische Eigenschaften usw., a. a. 0. 
Abzug der an sich auffällig starken glykolytischen Wirkung des letzteren keine deutliche Beeinflussung erkennen ließ.

\section{Versuche an Rückenmarksbrei.}

Die engen Beziehungen, die sich aus den vorangehenden Versuchen $\mathbf{z w i s c h e n ~ O r g a n f u n k t i o n ~ u n d ~ Z u c k e r u m s a t z ~ e r g e b e n ~}$ hatten, ließen erwarten, daß eine Zerstörung der normalen Struktur eine hochgradige Herabsetzung, wenn nicht Aufhebung des Zuckerverbrauches zur Folge haben würde. Um so überraschender erschien das Versuchsergebnis, da $B$ ein Zerreiben des Rückenmarks zu einem Brei keine Verminderung, sondern im Gegenteil sogar eine beträchtliche Steigerung des Zuckerverbrauches in Zucker-Kochsalzlösung nach sich zog.

Methodik. Das bei diesen Versuchen eingeschlagene Verfahren war das folgende: Die in der gewöhnlichen Weise präparierten und von ihrer Gefäßhaut befreiten Rückenmarke wurden nach erfolgter Wägung in einem Glasmörser soryfältig zerrieben und dann mit einer entsprechenden Menge Zuckerlösung (meist $6-10 \mathrm{ccm}$ ) in das Versuchsgefäß übergespült. Einige Schwierigkeiten bereitete das Durchleiten von Sauerstoff, da dieses in der etwas eiweißhaltigen Flüssigkeit ein Schäumen verursacht, durch welches Organpartikelchen an der Gefäßwand hinaufgeschoben und so aus der Zuckerlösung entfernt werden. Zur möglichsten Einschränkung dieses Übelstandes wurde der Gewebsbrei nach Überspülung in das Versuchsgefäß zunächst etwa 10 Minuten in diesem zentrifugiert, um ein Absetzen der Organpartikel am Boden zu bewirken, und der Sauerstoff wurde dann nicht wie sonst durch ein mit Watte verschlossenes Glasrohr in einem Strom feinster Bläschen, sondern durch ein ziemlich weites, offenes und nicht bis ganz an den Boden des Gefäßes reichendes Rohr in langsamen großen Gasblasen durchgeleitet. Gleichwohl ließ sich auch so ein geringer Substanzverlust nicht immer vermeiden, sỏdaß die Genauigkeit der Umrechnung auf die Einheit des Organgewichtes darunter litt. Zur Überführung in eine neue Lösung wurde der Gewebsbrei zunächst wieder 
scharf abzentrifugiert und die überstehende Flüssigkeit schnell abgegossen. Diese konnte auch nach Filtrieren wegen ihres Gehaltes an gelöstem Eiweiß oder an feinen durch das Filter gegangenen Partikelchen nicht ohne weiteres zur Zuckerbestimmung verwendet werden, sondern wurde erst nach dem von Michaelis ${ }^{1}$ ) empfohlenen Verfahren mit Liquor ferri oxydati dialysati enteiweißt.

Der auf diese Weise untersuchte Gewebsbrei zeigte, wie schon oben erwähnt, in einer $0,6 \% \mathrm{NaCl}$ und $0,5 \%$ Traubenzucker enthaltenden Lösung einen viel größeren Zuckerverbrauch als das intakte Organ, im Mittel von 7 Versuchen 7,4 mg $(5,8-9,5)$ pro $1 \mathrm{~g}$ und 24 Stunden. Auch an ein und denselben Präparaten ließ sich diese Steigerung des Zuckerumsatzes durch Zerreiben des Organs nachweisen: Zwei Rückenmarke, die in intaktem Zustande in den ersten 8 Stunden 5,2 mg Zucker (bezogen auf $1 \mathrm{~g}$ und 24 Stunden) verbraucht hatten, verbrauchten in den darauffolgenden 16 Stunden nach Zerreibung zu einem Brei 7,7 mg pro $1 \mathrm{~g}$ und 24 Stunden. Vorheriges Eintauchen des Rückenmarks in kochende Lösung beseitigte die Glykolyse gänzlich. Das nach Abfiltrieren des Breies gewonnene Filtrat zeigte auch in langdauernden Versuchen nur einen minimalen Zuckerverbrauch, der zweifellos von den durch das Filter gegangenen, zum Teil mit freiem Auge noch wahrnembaren Patikelchen herrührte; tatsächlich fehlte in einem durch 40 Stunden fortgesetzten Versuch, in welchem statt durch Papier durch ein dichtes Asbestfilter filtriert worden war, jegliche Glykolyse. Diese rührt also weder von Bakterien noch von einem löslichen Enzym her, sondern ist an die lebenden Gewebspartikel gebunden (womit jedoch die Möglichkeit einer Gewinnung intrazellularer glykolytischer Enzyme nach Art der Zymase nicht in Abrede gestellt werden soll).

Die im vorangehenden untersuchten Faktoren beeinflußten die Glykolyse des Rückenmarkbreies in ganz analoger Weise wie die des intakten Organs. So verminderte Sauerstoffmangel (7 stündiges Durchleiten von Stickstoff) in einem

3) L. Michaelis, Eine Mikroanalyse des Zuckers im Blut. Biochem. Zeitschr., Bd. 59, S. 166 (1914).

Hoppe-Seyler's Zeitschrift f. physiol. Chemie. C. 
Versuche den Zuckerverbrauch auf $4,1 \mathrm{mg}$ pro $1 \mathrm{~g}$ und 24 Stunden und dieser zeigte ebenso wie beim unversehrten Organ auch bei nachträglichem Sauerstoffdurchleiten (durch 17 Stunden) keine Erhöhung, sodá $B$ auch hier eine irreparable Schüdigung eingetreten war. Alkohol, in einer Konzentration von 5 Vol.-Proz. zu der gewöhnlichen Lösung zugesetzt, verminderte in einem Versuche den durch 11 Stunden untersuchten Zuckerumsatz auf $3,4 \mathrm{mg}$, während in den darauffolgenden 12 Stunden in alkoholfreier Lösung der normale Wert von $7,0 \mathrm{mg}$ wieder erreicht wurde. - Der Zusatz von $0,1 \%$ Chlorcalcium setzte den Zuckerverbrauch in einem durch 24 Stunden fortgesetzten Versuch aut $3,5 \mathrm{mg}$ herab; in einem zweiten Versuch verbrauchte der Rückenmarksbrei in den ersten 9 Stunden bei Calciumzusatz $2,7 \mathrm{mg}$, in den darauffolgenden 15 Stunden in calciumfreier Lösung $6,1 \mathrm{mg}$ pro $1 \mathrm{~g}$ und 24 Stunden. - Wie beim intakten Organ vermindert also auch beim Brei Narkotikum- und Calciumzusatz den Zuckerverbrauch hochgradig in mehr minder völlig reversibler Weise. Desgleichen hatte das Fehlen des Chlornatriums in der Zuckerlösung einen deutlichen schädigenden Einfluß auf die Glykolyse, wenn auch nicht so ausgesprochen wie beim ganzen Organ.

Über die Wirkung höherer Zuckerkonzentrationen $(1-2 \%)$ konnte auch am Brei kein eindeutiges Ergebnis gewonnen werden; im allgemeinen war der Zuckerumsatz in den konzentrierteren Lösungen von ungefähr gleicher Größe wie in der zu allen sonstigen Versuchen verwendeten $0,5 \%$ igen und zeigte auch hier wie am ganzen Organ eine Tendenz zum Ansteigen bei längerer Dauer.

Ein deutlich abweichendes Verhalten hingegen zeigte der Rückenmarksbrei gegenüber der elektrischen Reizung, die hier ganz ohne Einfluß blieb, wie die beiden folgenden Versuche dartun: Der durch Zerreiben zweier Rückenmarke gewonnene Brei verbrauchte in der ersten 15 stündigen Ruheperiode 5,8, in der darauffolgenden 8stündigen Reizungsperiode (Reizung 71/2 Stunden lang mit 30 tetanisierenden Reizen in der Minute in der gleichen Weise wie früher am intakten Organ) 
4,2 und in der daran wieder angeschlossenen 18 stündigen Ruheperiode $4,3 \mathrm{mg}$ pro $1 \mathrm{~g}$ und 24 Stunden. In ganz analoger Weise betrug in einem zweiten Versuch der auf $1 \mathrm{~g}$ und 24 Stunden bezogene Zuckerverbrauch zweier zerriebenen Rückenmarke in der ersten 15 stündigen Ruheperiode $6,1 \mathrm{mg}$, in der darauffolgenden 9 stündigen Reizungsperiode (Reizung 8 Stunden lang in der gleichen Weise wie in dem vorangehenden Versuch) 5,6 und in der letzten 16 stündigen Ruheperiode $5,0 \mathrm{mg}$. Die den Zuckerverbrauch steigernde Wirkung der Reizung ist mithin gebunden an die Funktionstätigkeit des intakten Organs.

\section{Erörterung der Versuchsergebnisse.}

Die im vorangehenden zusammengestellten Versuche haben dargetan, daß die Gewebe der nervösen Zentralorgane in der umgebenden Lösung eine nicht unbeträchtliche Menge Zucker zum Verschwinden bringen. Das Schicksal dieses Zuckers ist vorerst unbekannt. Die starke Beeinflussung der Glykolyse durch die Sauerstoffzufuhr, sowie die weitgehende Übereinstimmung in der Beeinflussung des Sauerstoffverbrauches und des Zuckerverbrauches durch verschiedenartige Faktoren machen es wahrscheinlich, daß der verschwindende Zucker in letzter Linie der Oxydation verfällt.

Sehr auffällig erscheint, die Beobachtung, daß das Rückenmark in zerriebenem Zustande einen größeren Zuckerumsatz aufweist als in normalem. Um festzustellen, ob auch hier eine Übereinstimmung in dem Verhalten von Zucker- und Sauerstoffverbrauch besteht, wurde in einigen Versuchen mit dem Mikrorespirationsapparat ${ }^{1}$ ) die Sauerstoffaufnahme des zerriebenen Rückenmarks untersucht; es zeigte sich aber, daß diese nicht bloß nicht höher, sondern bei weitem niedriger war als die des intakten Organs und nur etwa $1 / 3$ des normalen

') H. Winterstein, Ein Apparat zur Mikroblutgasanalyse und Mikrorespirometrie. Biochem. Zeitschr., Bd. 46, S. 440 (1912). - Ein Mikrorespiriationsapparat. Zeitschr. f. biol. Technik und Methodik, Bd. 3, S. 246 (1913). 
Wertes betrug. Während also die Oxydationsprozesse durch die Zerstörung der normalen Gewebsstruktur hochgradig geschädigt werden, erfährt die Zuckerzersetzung eine ansehnliche Steigerung. Bei dem Versuche einer Deutung dieser Erscheinung wird man zunächst $\mathrm{zu}$ berücksichtigen haben, daß die am intakten Organ gefundenen Sauerstoffwerte den physiologischen Verhältnissen weit eher entsprechen dürften als die Zuckerwerte. Denn während der Sauerstoff aus einer reinen Sauerstoffatmosphäre wohl mit genügender Schnelligkeit einzudiffundieren vermag, ist dies vielleicht beim Traubenzucker nicht der Fall. Und so erscheint als die nächstliegende Annahme die, daß es einfach die Vergrößerung der Kontaktfläche zwischen Gewebe und Zuckerlösung ist, welche die Verstärkung der Glykolyse beim Rückenmarksbrei verursacht. Unter physiologischen Bedingungen, wenn der Zucker den Zellen durch das Blut direkt zugeführt wird, könnte sein Umsatz im intakten Organ sehr wohl noch erheblich größer sein.

Jedenfalls zeigt schon dieses abweichende Verhalten des zerriebenen Rückenmarks hinsichtlich seines Sauerstoff- und seines Zuckerverbrauches, daß es sich keineswegs um eine direkte Oxydation des Zuckers handeln kann, daß vielmehr, wie ja auch schon von chemischen Gesichtspunkten aus wahrscheinlich ist, ein stufenweiser Abbau erfolgen dürfte, bei welchem die Oxydation erst in einer späteren Phase eingreift. In gleichem Sinne spricht auch die Beobachtung, daB Sauerstoffmangel keine Aufhebung, sondern nur eine Einschränkung des Zuckerverbrauches bedingt, die vielleicht als Reaktionshemmung durch Ansammlung sonst wegoxydierter Reaktionsprodukte verständlich erscheint.

Angesichts der durchaus gleichsinnigen Beeinflussung von Zucker- und Sauerstoffverbrauch durch NaCl-Mangel, Calciumzusatz und Urethannarkose erscheint ferner das gegensätzliche Verhalten der beiden Stoffwechselprozesse gegen Alkohol sehr befremdlich. Zu seinem Verständnis dürften vielleicht die folgenden Erwägungen beitragen: Wenn der Alkohol im Gegensatz zu anderen Narkoticis keine Herabsetzung, sondern sogar eine leichte Steigerung des Sauerstoffverbrauches ver- 
anlaßt, so ist diese Erscheinung wohl am einfachsten durch die Annahme erklärbar, daß der Alko hol zum Teil der Oxydation verfällt, und daß die hierzu erforderlicheSauerstoffaufnahme die übrige Einschränkung der 0xydationsprozesse wieder wettmacht, bezw. noch überkompensiert. Will man nun nicht die Annahme machen, daß gerade die der Alkoholoxydation zugrunde liegenden Vorgänge eine besondere Resistenz gegen Narkose aufweisen, so ergibt sich als wahrscheinlichste Vorstellung, daß es zunächst gar nicht die Oxydationsprozesse sind, welche durch die Narkose eine Hemmung erfahren, sondern die primären Spaltungsvorgänge, und daß erst sekundär infolge Ausbleibens der letzteren auch die Oxydation der Spaltungsprodukte in Fortfall kommt. In der Tat wurde schon bei der kritischen Erörterung der Erstickungstheorie ${ }^{1}$ ) eine Reihe von Tatsachen zusammengestellt, welche zeigen, daß die Oxydationsprozesse keineswegs zuerst, sondern vielfach erst bei bedeutend höheren Konzentrationen durch die Narkose gelähmt werden als andere Lebensvorgänge. Mithin läßt sich auch die Wirkung des Alkohols mit der Vorstellung primärer Spaltungen und sekundärer $0 x y-$ dation wohl in Einklang bringen.

Im Gegensatz zu allen übrigen Faktoren ist die Wirkung der elektrischen Reizung bloß am ganzen Organ und nicht am Gewebsbrei nachweisbar. Wir dürfen also daraus folgern, daß die durch sie bedingte Steigerung des Zuckerverbrauches erst die sekundäre Folge der an die Intaktheit des Organs gebundenen Erregungsvorgänge darstellt. In ganz analoger Weise wurde früher $^{2}$ ) der Nachweis geliefert, daß die elektrische Reizung nur insoweit eine Erhöhung des Sauerstoffverbrauches herbeiführt, als Erregungsprozesse ablaufen.

Die mit den Erregungsvorgängen verbundene, noch weit über die Erhöhung des Sauerstoffverbrauches hinausgehende Steigerung des Zuckerumsatzes ist sicher in hohem Maße bemerkenswert. Sie zeigt, daß der Zucker nicht bloß eine

1) H. Winterstein, Beiträge zur Kenntnis der Narkose. I. Mitt. Kritische Übersicht usw. Biochem. Zeitschr., Bd. 51, S. 143 (1913).

s) H. Winterstein, Beiträge zur Kenntnis der Narkose. II. Mitt. Biochem. Zeitschr., Bd. 61, S. 81 (1914). 
202 E. Hirschberg u. H. Winterstein, Über Zuckerstoffwechsel usw.

Quelle der Muskelkraft, sondern auch eine solche der Tätigkeit der nervösen Zentralorgane darstellt.

\section{Zusammenfassung.}

Das isolierte Froschrückenmark bewirkt in einer umgebenden Traubenzucker enthaltenden Lösung eine Glykolyse. Solange das Rückenmark von seiner Gefäßhaut umhüllt ist, ist der Zuckerverbrauch wegen der Undurchgängigkeit der letzteren minimal; nach Entfernung der Gefäßhaut beträgt er bei gewöhnlicher Zimmertemperatur in einer 0,2\% Zucker enthaltenden Lösung im Mittel $4 \mathrm{mg}$, in einer 0,5\% igen Zuckerlösung im Mittel $5 \mathrm{mg}$ pro $1 \mathrm{~g}$ und 24 Stunden.

Der Zuckerumsatz, dessen Größe von der Temperatur abhängig ist, nimmt mit der Versuchsdauer ab und ist am dritten Tage nicht mehr mit Sicherheit nachweisbar. Sauerstoffmangel bewirkt eine starke irreparable Herabsetzung desselben. Urethan und Alkohol in narkotischer Konzentration vermindern den Zuckerverbrauch hochgradig in reversibler Weise; das Gleiche gilt für den Zusatz von Calciumsalzen schon in geringen Mengen $\left(0,1 \% \mathrm{CaCl}_{2}\right)$. Fehlen des Chlornatriums drückt, vermutlich vor allem wegen der starken Quellung, die Glykolyse auf ein Minimum herab. Elektrische Reizung ruft eine außerordentliche starke Steigerung des Zuckerverbrauches hervor, der auf mehr als das Doppelte des Ruhewertes ansteigen kann.

Die zu Brei zerriebene Rückenmarksubstanz zeigt, vermutlich infolge Vergrößerung der Kontaktfläche zwischen Gewebe und Zuckerlösung, eine im Mittel um etwa 50\% höhere Glykolyse als das intakte Organ. Diese Glykolyse ist an die lebende Zellsubstanz gebunden; im Brei des gekochten Organs sowie in dem von Gewebspartikeln freien Filtrat ist sie nicht nachweisbar. Der Zuckerbrauch des Rückenmarkbreies wird durch die oben genannten Faktoren im gleichen Sinne beeinflußt wie der des intakten Organs, mit der Ausnahme, daß die elektrische Reizung nach Zerstörung der normalen Struktur keine Steigerung des Zuckerumsatzes hervorruft. 